\title{
Recurrent sub-mucosal dissection of the oesophagus in association with achalasia
}

\author{
T.L. Hooper, J. Gholkar, S.R. Smith, J.J. Manns and H. Moussalli \\ Wythenshawe Hospital, Southmoor Road, Manchester M23 9LT, UK.
}

\begin{abstract}
Summary: Submucosal dissection of the oesophagus is a rare oesophageal disorder. We report a patient who had recurrent episodes of dissection and achalasia. Both recurrence and the association with achalasia are to our knowledge unique.
\end{abstract}

\section{Introduction}

Spontaneous dissecting intra-mural haematoma is a rare oesophageal disorder first described by Williams (1957). In a recent review only 28 further cases had been reported in the literature (Spiller et al., 1981), and the benign course and non-recurrent nature of the condition was emphasized. We describe a unique case which was recurrent and occurred in association with achalasia, which may have aetiological implications.

\section{Case history}

A 45 year old woman presented with a 30 -hour history of a sudden onset of right sided chest pain radiating to her back. Several hours later she began to vomit, initially clear fluid, but subsequently fresh blood. She gave no history of alcohol excess or of a recent heavy meal. Two years previously she had undergone oversewing of a perforated duodenal ulcer, and 19 years before, a Heller's operation had been carried out via an abdominal approach.

Physical examination revealed a pyrexia of $37.8^{\circ} \mathrm{C}$ but was otherwise unremarkable. Full blood count, blood urea, electrolytes and amylase were normal as were coagulation studies. Chest and abdominal radiographs were also normal. Barium swallow demonstrated a dilated oesophagus with tertiary contractions, tapering to a smooth narrowing at its lower end consistent with achalasia. In addition it revealed a 'double lumen' appearance of the mid and lower oesophagus with a visible mucosal partition, suggesting an intra-mural dissection (Figure 1). Oesophagoscopy confirmed a free flap of bleeding mucosa at $30 \mathrm{~cm}$ from the incisors, beyond which the mucosa bulged into the lumen preventing further passage of the oesophagoscope.

Correspondence: T.L. Hooper, F.R.C.S.

Accepted: 12 May 1986
Treatment was conservative with nothing by mouth for 10 days, following which a repeat barium swallow was performed which no longer demonstrated the dissection. Diet was re-commenced and further recovery was uneventful. At follow-up she remained symptom-free and swallowing normally.

Fifteen months later she presented again with a very similar history though on this occasion the pain preceded the vomiting by 12 hours. Barium swallow showed a further extensive sub-mucosal dissection (Figure 2), and endoscopy revealed a sub-mucosal haematoma partially occluding the lumen and extending from 18 to $32 \mathrm{~cm}$. On this occasion a free flap of mucosa was seen at the lower end of the haematoma. Once again her symptoms resolved with conservative measures, and she remains well 6 months later.

\section{Comment}

Two types of spontaneous injury of the oesophagus are well recognized. The Mallory-Weiss tear is a mucosal laceration occurring at the oesophago-gastric junction and most commonly follows vomiting (Graham \& Schwarz, 1977). Trans-mural rupture (Boerhaave's syndrome) also occurs close to the diaphragm or sometimes in the mid oesophagus, is not necessarily related to vomiting, and carries a high mortality (Walker et al., 1985). A dissecting intra-mural tear is a much rarer and less well known injury. It affects adults of all ages, and typically presents with severe chest pain radiating to the back, associated with haematemesis and occasionally acute dysphagia (Spiller et al., 1981). A preceding history of vomiting or retching is not common, though the event may follow such apparently trivial insults to the oesophagus as drinking a glass of beer or swallowing a meal hurriedly. The radiological and endoscopic findings reported here are typical, as is the relatively benign

(C) The Fellowship of Postgraduate Medicine, 1986 


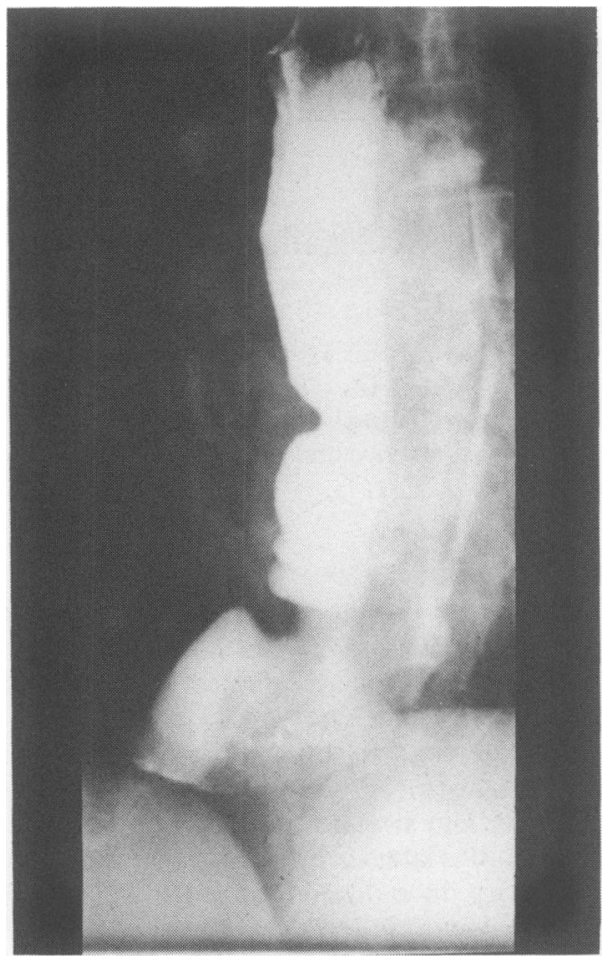

Figure 1 Barium swallow showing features of achalasia and a 'double lumen' appearance, suggesting a submucosal dissection.

course and the success of conservative treatment.

The aetiology of the disorder is unknown. It has been suggested that the intra-mural bleed predates the occurrence of the mucosal tear, resulting in a submucosal collection of blood such as occurs following trauma from swallowed foreign bodies or endoscopic procedures (Smith et al., 1974). The sub-mucosal plane would offer little resistance to an expanding haematoma, and may account for the extensive dissections seen here. A mucosal tear would then decompress the haematoma and result in haematemesis. Our case would support this hypothesis, firstly because pain preceded vomiting by several hours, and clear fluid was produced before any blood, and secondly,

\section{References}

GRAHAM, D.Y. \& SCHWARZ, J.T. (1977). The spectrum of the Mallory-Weiss tear. Medicine, 57, 307.

SMITH, G., BRUNNEN, P.L., GILLANDERS, L.A. \& TEO, H.S. (1974). Oesophageal apoplexy. Lancet, i, 390.

SPILLER, R.C., CATTO, J.V.F. \& KANE, S.P. (1981). Spontaneous dissecting intra-mural haematoma of the oesophagus: a rare cause of haematemesis and dysphagia. Endoscopy, 13, 128.

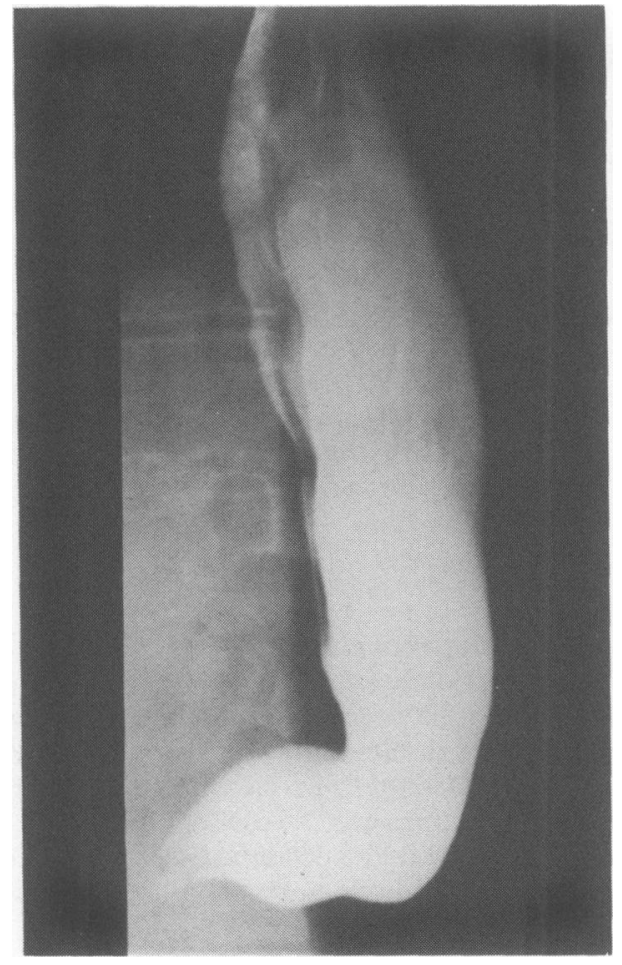

Figure 2 Fifteen months later, a further dissection, but at a different site.

because of the position of the mucosal tear in the first instance at the upper limit of the haematoma and on the second occasion at its lower extent. The reason for a spontaneous intra-mural haemorrhage is not known; defects of coagulation are not found in the majority of cases (Spiller et al., 1981).

There has been no previously reported case of a recurrent submucosal dissection, nor has an association with achalasia been described. It is possible that disordered oesophageal function may in some way predispose to this condition. Indeed pre-existing hiatus hernia and reflux oesophagitis have been noted in some cases (Smith et al., 1974).

WALKER, W.S., CAMERON, E.W.J.\& WALBAUM, P.R. (1985), Diagnosis and management of spontaneous transmural rupture of the oesophagus (Boerhaave's syndrome). British Journal of Surgery, 72, 204.

WILLIAMS, B. (1957). Oesophageal laceration following remote trauma. British Journal of Radiology, 30, 666. 